\title{
THE DIAGNOSIS AND TREATMENT OF THE FRACTURED SPINE IN THE LUMBODORSAL REGION.
}

\author{
By ALAN C. GAIRDNER, B.M., F.R.C.S. \\ (Hon. Assistant Surgeon to the Royal Devon and Exeter Hospital.)
}

It has until recent years been the custom to regard the fractured spine as important only in its neurological aspect, or, in other words, that fracture of the spine is a serious matter only if complicated by some damage to the spinal cord.

If a series of cases of fractured spine is analysed, it is found that in only a very small proportion does injury to the spinal cord occur. Nevertheless, of those without cord injury, if they are treated by simple recumbency, $70 \%$ remain permanently disabled from pain and stiffness in the back. Moreover, in a certain number pain develops in the back, although at the time of the accident no fracture was diagnosed, but merely a slight deformity of a vertebral body. These latter patients develop a very intractable disability, consisting of pains in the back more or less severe, which tends to persist through life.

It would therefore appear that while the nervous damage is the more dramatic and the more serious injury, simple bony damage is a matter of very grave import, leading to prolonged disability, even if the original deformity is not of any very great severity. This is undoubtedly due to the presence of a localised spondylitis which can be seen developing in a consecutive series of radiograms. The cause of this sequela is easily apparent if we consider that a fracture of the body of a vertebra is not a simple fracture but one which almost always involves an intervertebral joint. Should consolidation of the fracture occur with the deformity still present, movement around the damaged joint will inevitably, as in all situations, produce an osteoarthritis which will continue with increasing severity as long as the joint is in movement.

It is found that under two circumstances only does a painless back result after a fracture:-

I. Where the deformity is completely reduced.

2. Where local fixation of the deformity by bone has occurred.

In either case a sound painless and useful back is obtained.

It would therefore appear that treatment must be directed to obtain one of these two results, either a mobile spine without deformity, or a locally fixed spine with more or less deformity.

From the above considerations it is clear that it is of the greatest importance to diagnose accurately the presence of any fracture of a vertebral body, even if the deformity is very slight, since a very minor maladjustment tends to produce later severe disability.

Where the injury is very severe the diagnosis will not present much difficulty, as the presence of a kyphotic deformity with tenderness and rigidity of the spine combined with the radiogram will always indicate a gross injury. If, however, the deformity is slight and consists of a minor wedging of one vertebral body, the diagnosis is more difficult as no kyphosis is apparent on examination and the patient simply has pain and rigidity in the spine, symptoms which may easily be produced by a severe sprain. It must be borne in mind, too, that an anteroposterior radiogram does not reveal minor wedge deformities of a vertebral body 
owing to the fact that the height of the posterior surface of the body of the vertebra is not diminished and the view obtained by $\mathrm{X}$-ray shows only this undiminished view. A lateral view is therefore essential in order to show minor deformities. A second point which makes diagnosis by the X-rays difficult, is the fact that men who are habitually engaged in heavy work, particularly in heavy carrying after the age of 35 , almost invariably show a marked wedging of the I2th dorsal and Ist lumbar vertebræ which in later years is frequently accentuated by spondylitis. In such patients the radiogram often reveals a very marked wedging with irregularity which may be mistaken for a fracture.

Where doubt exists as to whether a fracture is present or not the X-ray findings must be taken in conjunction with the physical signs.

The diagnosis of fracture can usually be made by the presence of the following points :-

I. Radiogram showing wedging of a vertebra with some irregularity and discontinuity of the solid bony anterior shell.

2. Tenderness localised over the spinous process of the suspected vertebra.

3. Pain localised at the suspected site on pushing down on the shoulder.

4. Pain marked and localised in bending the body forward (thus compressing the fractured area) and more marked than on posterior or lateral bending.

Should these physical signs be present, deformity of the body seen in the radiogram is almost certainly a fracture of recent date and not of long standing.

\section{Treatment.}

As has been previously indicated the treatment of the fractured spine must be directed to the attainment of a good functional and painless back. This may be obtained in one of two ways, either by the complete reduction and consolidation of the deformity or by the local fixation of the spine in a more or less deformed position. There is no question that of the two the first is the more desirable, as a normal anatomy is secured and it is to the attainment of this end that treatment should at first be directed.

Fixation by bone graft should be held in reserve until the first method has been tried and has failed.

To restore the deformity it is necessary to understand thoroughly its nature firstly, and the force which has produced it.

The deformity in almost all cases of fracture is a deformity of the anterior part of the body of the vertebra which appears in this way.

ANT.

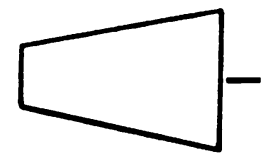

POST.

The body is forced into a wedge shape which is caused by a forcible bending forward of the spine. In this way the posterior part of the body escapes the main 
injury, and the deformity is usually confined to the anterior portion of the vertebra. In severe injuries, however, two further conditions may be seen. (I) The anterior triangular portion of the body is forced forward and (2) the posterior portion is squeezed and forced back giving an associated dislocation as shown in the following diagrams.
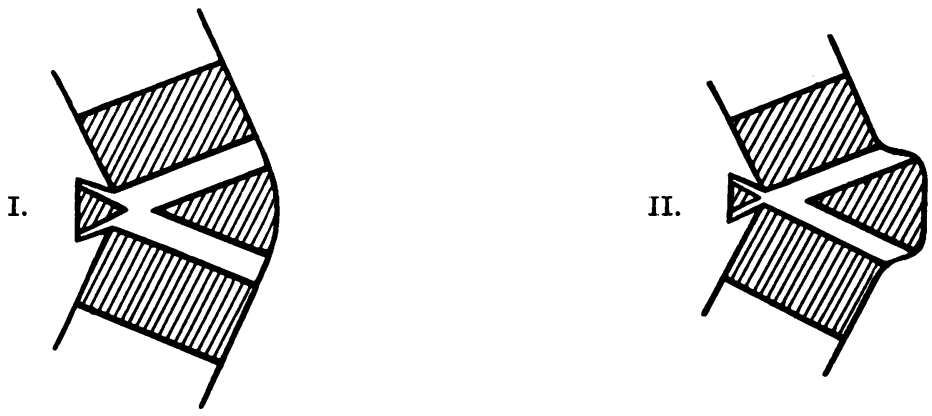

Evidently, therefore, the method of reduction which would logically be of most value is hyperextension. This produces traction on the anterior and posterio common ligaments and on the intervertebral discs, and tends to reduc the deformities. It has been shown by various authors that reduction of the deformity can be attained in this way. Various methods have been tried, either gradual hyperextension on a special bed, or immediate hyperextension with fixation by Plaster of Paris: of the two the second is the more practicable and more comfortable for the patient.

\section{Method of Reduction Described.}

Many methods have been described, but the following has been found to give good results in the hands of the writer. On arrival in hospital with a suspected fractured spine the patient is nursed on his face. When the diagnosis is confirmed the patient is fully anæsthetised and placed face downwards on a canvas sling in an Abbott's Frame. A hole is previously made in the sling through which the patient's face projects for anæsthesia. Gaiters are then attached to the patient's ankles and the feet are drawn up so that the back is very fully hyperextended. The weight of the patient's head and upper chest is carried by the canvas sling, but the pelvis is held just clear of the sling. The patient having thus been hyperextended, firm and sudden pressure is exerted with both hands over 9 the region of the fracture in order to disimpact it. A grating click may often be $\frac{D}{O}$ obtained. A plaster is then applied in the hyperextended position and stretches in front from the sternal notch to the pubes below, and behind from about the third o thoracic spine to the middle of the sacrum. For comfort the plaster may be $N$ cut away in the axillæ and need only be relatively short behind but it must be long in front in order to preserve the hyperextended position. The one weak spot in 9 this jacket is the arch of the back behind, and consequently this must be strengthened by many plaster strips. 
The patient is then removed to the ward in the supine position, a pillow being packed in the hollow of the plaster to prevent cracking.

\section{After-Treatment.}

As can be imagined the treatment may lead to a certain amount of shock and the patient should be treated accordingly. On recovery from the anæsthetic the plaster is usually well tolerated and is found to have abolished completely any pain at the site of the fracture, so that the patient can be nursed with ease and moved freely in all directions without pain.

There is, however, one serious but relatively rare complication, viz.:- acute dilatation of the stomach. Vomiting occurs more or less constantly after the application of the plaster. This if not treated may lead to an acute dilatation of the stomach, which is probably due to an obstruction of the duodenum by the superior mesenteric vessels, as in the hyperextended position the small intestine tends to gravitate into the pelvis and pull on the superior mesenteric vessels, while the duodenum is thrust forward by the second lumbar vertebra which forms the highest point of the arch in the hyperextended spine. This was clearly shown at postmortem examination in one of the author's cases (L.C.C. Medical Reports I933), the cause of death being acute dilatation of the stomach.

In order to avoid this complication the patient 8 hours after the application of the plaster, when it has usually set, is nursed for half of the 24 hours either prone or on the side. The assumption of this posture usually causes the vomiting to stop. If, however, this manœuvre is not successful the patient must be nursed entirely prone and the stomach washed out every 4 hours. If vomiting continues and the patient deteriorates rapidly, the plaster should be removed as a life saving measure.

If all goes well the patient is allowed to sit up after 2-3 weeks, and if able may walk after about 6 weeks. The plaster is retained for 4 months when a spinal jacket of the Ernst type with axillary crutches is fitted. This should be worn for a further 8 months. Consolidation of the fracture should have occurred by this time.

If, as a result of the treatment, perfect restitution has been obtained it is probable that no further trouble will ensue. If, on the other hand, there is imperfect reduction, or there is joint damage such as nuclear prolapse, a localised spondylitis will sooner or later supervene. This leads to a very marked crippling of the back owing to severe pain and limitation of movement. In these circumstances there is no question that fixation of the affected vertebræ is the treatment of choice, either by Albee or Hibbs method and this treatment has produced many excellent results.

\section{Conclusions.}

If the fractured spine is treated thoroughly by one of the two methods described above, a result approximating to Ioo per cent. cure should be obtained in all cases in which there is not any permanent nervous damage.

The presence of nervous damage in the early stages is no contraindication to hyperextension treatment. Indeed, it is particularly indicated in such cases, since hyperextension tends to restore the normal contour of the spinal canal in a less dangerous and more anatomical manner than can be obtained by laminectomy. Laminectomy, if anything, further weakens the spine and tends to perpetuate the deformity, and consequently when this operation is indicated it probably would be desirable to combine it with a double bridge bone graft after the manner described by Girdlestone for compression paraplegia in caries of the spine. 